\title{
Controlled-release microchip
}

Researchers at the Massachusetts Institute of Technology have developed a microchip that could one day allow the automated delivery of drugs such as insulin. In a recent issue of

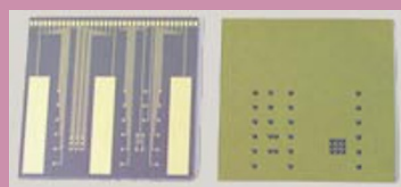
Nature (397, 335-338, 1999), Robert Langer and his colleagues at MIT (Cambridge, MA) report the creation of a chip etched with reservoirs that can be loaded with liquid and "opened" using electrical current. Each reservoir is gated by a gold membrane that dissolves when an electrical current passes through it, allowing the controlled release of the reservoir contents. At their present size, microchips can accommodate approximately 1,000 such reservoirs. Langer believes that this technology could be coupled to a microprocessor and a battery to allow the timed administration of powerful drugs such as painkillers, chemotherapeutic agents, and fertility drugs over prolonged periods. "This could be a biocompatible, self-contained unit, possibly swallowable," he envisages. In addition to drug delivery, the microchips could also be used in fields such as medical diagnostics, chemical detection, and microbiology. As with any emerging technology, Langer is excited by the possibilities: "There are so many other applications, right now, it's hard to predict where it will end up."

\section{Muscling in on bone formation}

Addressing the National Meeting of the Orthopedic Research Society in Anaheim, CA, at the beginning of February, Johnny Huard described work at the University of Pittsburgh and the Children's Hospital of Pittsburgh demonstrating the ability of muscle-derived stem cells to form bone when transplanted into mice. Huard and his colleagues accomplished this by isolating a special population of myogenic cells within skeletal muscle that appear capable of forming bone when stimulated in vitro with bone morphogenic protein BMP-2. To test whether these bone-forming cells could be induced experimentally, they then injected genetically altered musclederived stem cells expressing BMP-2 protein and $\beta$-galactosidase into the hindlimb muscles of mice. As predicted, the BMP-2-expressing cells were able to form ectopic bone in the muscle in vivo. As Huard explains: "The colocalization of $\beta$-galactosidase and the bone marker, osteocalcin, conclusively shows that these cells are not trapped, but active in bone formation." The findings may be clinically relevant to orthopedic patients suffering from severe bone fractures, as muscle-derived stem cells could be used to develop faster, more economical means of delivering osteoprogenitor cells directly to the wound site. Experiments are currently underway to investigate whether muscle stem cells are truly pluripotent.

Research News Briefs written by Aseem Answari, Alex Castellino, Margret Einarson, and LeeAnn Leshko.

\section{Reprogramming neural stem cells}

The notion that stem cell fate is dictated by the tissue of origin has been challenged by recent work published in Science (283, 534-537, 1999). In collaboration with colleagues from NeuroSpheres (Calgary, Alberta, Canada) and Istituto di Mutagenesi (Pisa, Italy), Angelo Vescovi of the Istituto Nazionale Neurologico Carlo Besta (Milan, Italy) have shown that neural stem cells (NSCs) can adopt hematopoietic identity when injected into mice. By tag-

ging the NSCs with lac $Z$, the authors were able to confirm that donor DNA was present in the

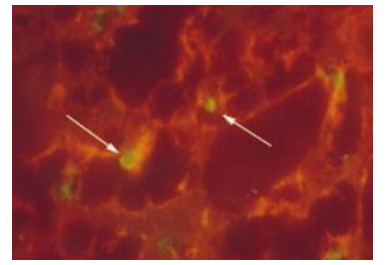
spleen of re-

cipient mice and that transplanted NSCs were capable of producing $\mathrm{B}$ cells, $\mathrm{T}$ cells, and myeloid cells. What's more, hematopoietic antigens were detected on these cells that were distinctly absent in NSCs before transplantation. According to Vescovi, "The inherent plasticity of highly undifferentiated stem cells is probably wider than what was originally thought. This allows them to respond to the environmental cues in which they find themselves." It remains to be determined whether human stem cells show the same plasticity and broad specificity as mouse NSCs. If the results prove comparable, large batches of stem cells for use in leukemia could be grown, providing an alternative to stem cells derived from human embryos, which pose ethical problems.

\section{Sizing up DNA}

Researchers at the California Institute of Technology have developed a novel microfabricated device that uses fluorescence detection to size and sort individual DNA molecules at a rate 100 -fold faster than conventional gel electrophoresis while requiring a million fold less sample. The device consists of an elastomer microfluidic network, which can be loaded with femtograms of DNA labeled with YOYO-1, an intercalating fluorescent dye. Illumination of the sample with lasers allows researchers to use fluorescence quantitatively to measure the length of individual DNA molecules. Lead investigator, Stephen Quake, explains that the total fluorescence intensity of the sample is directly proportional to the size of the DNA molecule. "We would ultimately like to use this device for nested mapping," he says. This would involve selecting and manipulating a DNA molecule into a specific channel, and then carrying out successive enzymatic reactions directly on the chip. Ultimately, the device could prove useful for bacterial artificial chromosome clone fingerprinting and DNA diagnostics. The findings are reported in Proc. Natl. Acad. Sci. USA $(96,11-13,1999)$.

\section{Priming axon regeneration}

In a recent report in Neuron (22, 89-101, 1999), Marie Filbin and her colleagues at the Hunter College of the City University of New York have elegantly shown how mature neurons can overcome growth inhibition imposed by myelin proteins, with significant implications for the treatment of spinal injuries. In healthy neurons, myelin is thought to provide cues essential for cell maintenance; when neurons are damaged, however, these signals appear to be misread, preventing neuronal regeneration. "There are several inhibitory factors in myelin, including the myelin-associated glycoprotein (MAG)," says Filbin. "We found that preincubating mature neurons with certain neurotrophins allows them to grow in the presence of MAG and, in fact, in the presence of all inhibitory factors in myelin." The researchers also showed that cAMP levels in mature neurons were enhanced by the neurotrophins. As a corollary, inhibiting the function of the protein kinase A, the cAMP-dependent kinase, abolished the ability of neurotrophins to override the growth-inhibitory myelin signals. Interestingly, myelin was also shown to trigger an inhibitory Gi protein in neurons. According to Filbin, the Gi signal prevents an increase in cAMP levels and consequently arrests nerve growth. 